to IFN-I production and subsequent induction of interferon-stimulated genes (ISGs).

Objectives: To explore the potential of TBK1 inhibitors to downregulate IFN-I activation in SLE and SSc.

Methods: TBK1, IRF3, IRF7 and STAT1 were determined by qPCR in PAXgene samples and phosphorylated-TBK1 (pTBK1) was analysed by flowcytometry in plasmacytoid dendritic cells ( $\mathrm{pDCs}$ ) from IFN-I positive (IFNpos) patients. Peripheral blood mononuclear cells (PBMCs) from SLE and SSc patients and TLR7stimulated PBMCs from healthy controls ( $\mathrm{HCs}$ ) were cultured with the TBK1 inhibitors BX795 followed by analysis of ISGs.

Results: Increased expression of TBK1, IRF3, IRF7 and STAT1 in whole blood and pTBK1 in pDCs was observed in IFNpos pSS, SLE and SSc patients compared to HCs. Upon treatment with BX795, PBMCs from IFNpos pSS, SLE, SSc and TLR7-stimulated HCs downregulated the expression of the ISGs MxA, IFI44, IFI44L, IFIT1 and IFIT3. The TBK1 inhibitor inhibited the secretion of IFN-I by TLR7-stimulated PBMCs from HCs.

Conclusions: TBK1 inhibition reduced expression of ISGs in PBMCs from IFNpos SLE and SSc patients patients indicating TBK1 as a potential treatment target.

Acknowledgements: The research for this manuscript was (partly) performed within the framework of the Erasmus Postgraduate School Molecular Medicine. The authors thank patients and healthy volunteers for taking part in this study.

Disclosure of Interest: E. Huijser: None declared, I. Bodewes: None declared, C. van Helden-Meeuwsen: None declared, L. Tas: None declared, R. Huizinga: None declared, V. Dalm: None declared, M. van Hagen: None declared, N. Groot: None declared, S. Kamphuis: None declared, P. van Daele: None declared, M. Versnel Grant/research support from: Research funding Domainex

DOI: 10.1136/annrheumdis-2018-eular.6367

\section{AB0039 IMMUNOSUPPRESSIVE EFFECT OF MESENCHYMAL STEM CELLS ON MONOCYTES DERIVED FROM PATIENTS WITH RHEUMATOID ARTHRITIS}

G. Vasilev $^{1}$, M.G. Ivanova ${ }^{1}$, E. Ivanova-Todorova ${ }^{1}$, K. Tumangelova-Yuzeir $^{1}$, E. Krassimirova ${ }^{1}$, R. Stoilov ${ }^{2}$, D. Kyurkchiev ${ }^{1} .{ }^{1}$ Laboratory of Clinical Immunology, University Hospital "St. Ivan Rilski" Sofia; ${ }^{2}$ Clinic of Rheumatology, University hospital "St. Ivan Rilski" Sofia, Medical University of Sofia, Sofia, Bulgaria

Background: Mesenchymal stem cells (MSCs) are a heterogeneous population of fibroblast-like progenitor cells that may be isolated and expanded from bone marrow, umbilical cordand other tissues. Moreover, MSCs display the capacity to self-renew and differentiate into various mesodermal cell lineages. They also have drawn considerable attention for their regenerative and immunosuppressive effects in a range of autoimmune diseases, which raises the question for their potential use as therapeutic tool in rheumatoid arthritis.

Objectives: In line with aforementioned and taking in mind that monocytes playing pivotal role in orchestrating immunological tolerance, we aimed to study the immunosuppressive effects of adipose tissue derived mesenchymal stem cells (AT-MSC) on monocytes derived from patients with rheumatoid arthritis (RA).

Methods: We enrolled 12 patients matching the ACR/EULAR 2010 criteria for RA. AT-MSC were isolated and cultured according to well established protocols. ELISA was performed for testing the cytokines produced by AT-MSC (IL-1, IL-10, IL-4, IFN- $\gamma$, IL-6, IL-8, CCL-5, RANTES, IL-17). Peripheral blood mononuclear cells (PBMCs) isolated from RA patients' samples were cultured in AT-MSC conditioned media and in control media. Flow cytometry was used for detection of monocytes markers (CD14, CD80, CD86, HLA-DR).

Results: Our results revealed considerable increase in number of cells expressing the myeloid lineage surface marker CD14 $(p=0.026)$ under the influence of AT-MSC medium. We further demonstrated decrease in percentage values of monocytes expressing HLA-DR $(p=0.004)$ and CD80/86 surface molecules $(p=0.47)$. Moreover, significant reduction in mean fluorescent intensity of HLA-DR surface expression $(\mathrm{p}=0.016)$ was also demonstrated.

Conclusions: Our study unambiguously points out that under the impact of ATMSC media monocyte development into dendritic cells was hampered and was also skewed into direction of accumulation of monocytes displaying more tolerogenic phenotype characterised by down regulation of HLA-DR and B7 complex (CD80/86) surface expression. Furthermore, antigen presentation alongside with expression of the co-stimilatory B7 complex and cytokine ambience forms a three-way signalling pathway responsible for $\mathrm{T}$ helper activation and proliferation and consequent antibody production by plasma cells. Hence, effects exerted by AT-MSC conditioned media on monocyte lineage development should not be neglected in the context of developing novel therapeutic approaches and strategies for treatment of RA.

Disclosure of Interest: None declared

DOI: 10.1136/annrheumdis-2018-eular.3201

\section{$\mathrm{AB} 0040$}

TOLL-LIKE RECEPTOR 9 INFLUENCES INFLAMMATORY ARTHRITIS AND OSTEOCLASTOGENESIS

A. Fischer ${ }^{1}$, S. Abdollahi-Rodsaz ${ }^{2,3}$, A.C.Y. Yau ${ }^{4}$, E. Lönnblom ${ }^{4}$, B. Meyer ${ }^{1}$ B. Niederreiter ${ }^{1}$, R. Holmdahl ${ }^{4}$, G. Steiner ${ }^{1} .{ }^{1}$ Division of Rheumatology, Internal Medicine III, Medical University of Vienna, Vienna, Austria; ${ }^{2}$ Department of Rheumatology, Radboud University Nijmegen Medical Centre, Nijmegen, Netherlands; ${ }^{3}$ Division of Rheumatology, Department of Medicine, New York University School of Medicine, New York, USA; ${ }^{4}$ Medical Inflammation Research, Department of Medical Biochemistry and Biophysics, Karolinska Institute, Stockholm, Sweden

Background: Release and insufficient removal of endogenous nucleic acids may be involved in triggering autoimmune reactions important in the initiation of systemic autoimmune diseases including rheumatoid arthritis (RA). Nucleic acid sensing molecules, such as the endosomal Toll-like receptors (TLRs) 7 and 9 have been linked to pathogenic autoimmune processes, but their role in RA is less clear. Data obtained in rats with pristane-induced arthritis (PIA) suggested involvement of TLR9 in the pathogenesis of this arthritis model ${ }^{1}$.

Objectives: To gain more insight into the role of TLR9 in autoimmune arthritis TLR9 inhibition with specific oligodeoxynucleotide sequences (ODN) ${ }^{2}$ was investigated in rats with PIA. To further investigate TLR9 involvement, streptococcal cell wall (SCW) arthritis was induced in $\mathrm{TLR9}^{-/}$mice.

Methods: Arthritis was induced in mice with SCW lysates and in rats with the mineral oil pristane. Rats were treated with a TLR9 antagonist, starting before disease induction. Arthritis was scored using established scoring systems, inflammation and bone erosion were quantified by histological analysis. Levels of $\alpha-1$-acid-glycoprotein (AGP), rheumatoid factor (RF) and IL- 6 in sera were analysed. The role of TLR9 in osteoclast differentiation and activation was investigated in vitro.

Results: In PIA, the TLR9 antagonist reduced arthritis severity by $\sim 50 \%$, which was accompanied by a reduction of AGP, IL- 6 and RF. TLR9 inhibition led to reduced inflammation, bone erosion and cartilage degradation. Moreover, the $T$ cell-dependent chronic phase of SCW arthritis was significantly suppressed in $\mathrm{TLR}^{-/-}$mice. Remarkably, TLR7 and TLR9 mRNA levels strongly differed in the course of in vitro osteoclastogenesis. Whereas TLR7 expression did not change throughout osteoclastogenesis, expression of TLR9 was higher in precursor cells than in mature osteoclasts and stimulation with a TLR9 agonist (CpG) completely inhibited osteoclastogenesis.

Conclusions: Taken together, the results suggest a role for TLR9 in the T celldependent phases of PIA and SCW arthritis thus an important involvement of the DNA (CpG) recognising TLR9 in the initiation of autoimmune arthritis and during osteoclastogenesis. The precise role(s) of TLR9 in the different stages of arthritis needs to be further elucidated in future experiments.

\section{REFERENCES :}

[1] Hoffmann $\mathrm{MH}$, et al. Nucleic acid-stimulated antigen-presenting cells trig ger $\mathrm{T}$ cells to induce disease in a rat transfer model of inflammatory arthritis. J Autoimmun, 2011;36(3-4):288-300.

[2] Duramad O, et al. Inhibitors of TLR-9 act on multiple cell subsets in mouse and man in vitro and prevent death in vivo from systemic inflammation. $J$ Immunol, 2005;174(9):5193-200.

Disclosure of Interest: None declared DOI: 10.1136/annrheumdis-2018-eular.4557

\begin{tabular}{|l|l|}
\hline AB0041 & HETEROGENEITY OF THE TYPE I INTERFERON \\
& SIGNATURE IN RHEUMATOID ARTHRITIS MAY \\
& ACCOUNT FOR DIFFERENCES IN ITS CLINICAL \\
& RELEVANCE
\end{tabular}

J. Rodríguez-Carrio $^{1,2}$, M. Alperi-López ${ }^{2,3}$, P. López ${ }^{1,2}$, F.J. Ballina-Garcia ${ }^{2,3}$, A. Suárez ${ }^{1,2}$. ${ }^{1}$ Area of Immunology, University of Oviedo; ${ }^{2}$ Instituto de Investigación Sanitaria del Principado de Asturias (ISPA); ${ }^{3}$ Department of Rheumatology, Hospital Universitario Central de Asturias, Oviedo, Spain

Background: increased expression of interferon-responding genes (IRGs), the so-called IFN signature, has been reported in rheumatoid arthritis (RA) in peripheral blood and synovial tissue. However, some controversy exists concerning its clinical relevance, since longitudinal changes of the IFN signature have been reported upon different treatments and a physiological diversification of the type I IFN signature among different conditions has been recently described. Additionally, the relevance of the IFN signature in the very early stage of RA is unknown. 
Objectives: to evaluate (i) whether quantitative and qualitative differences in the activation of the IFN signature can be found in RA patients depending on the clinical stage and (ii) if these differences may be linked to a clinical relevance of the IFN signature.

Methods: expression of IFI44, IFI44L, IFI6 and MX1 was determined in peripheral blood in 98 RA patients and 28 controls. RA patients were classified into groups according to their clinical stage and treatments received: very early RA (VERA, recruited at onset and not exposed to any treatment), bDMARD-naïve (patients on csDMARD treatment) and bDMARD (patients on biological treatment, all antiTNF $\alpha$ agents). An additional group of 13 RA patients candidate for TNF $\alpha$-blockade was also recruited and samples were taken before and after anti-TNF $\alpha$ treatment. The associations among IRGs were evaluated by network and principal component analyses.

Results: all IRGs was increased in RA, although differences were noted among them. The IFN score was increased in all RA groups (VERA, bDMARD-naïve and bDMARD), but differences in their degree of activation and in the relationships among IRGs were observed. VERA patients exhibited a lower activation of the IFN signature and a distinct picture of the structure of the IRG network (figure 1) compared to both their established disease-counterparts and the HC group. The IFN score correlated the accumulated DAS28 over one year $(r=0.593, p=0.025)$ and it was found to be a predictor of a good clinical outcome (EULAR good clinical response) in VERA ( $A \cup C=0.917, p=0.004$ ). However, no differences in the IFN score were observed between the bDMARD-naïve and bDMARD groups, but opposite associations with the clinical parameters were noted. Interestingly, the correlations among IRGs delineate different pictures between these two groups. The IFN score at baseline predicted poor clinical outcome upon TNF $\alpha$-blockade. Although no absolute changes in the IFN score were found, TNF $\alpha$-blockade shifted the associations among IRGs. These differences mirrored those found when comparing bDMARD-naïve and bDMARD groups.
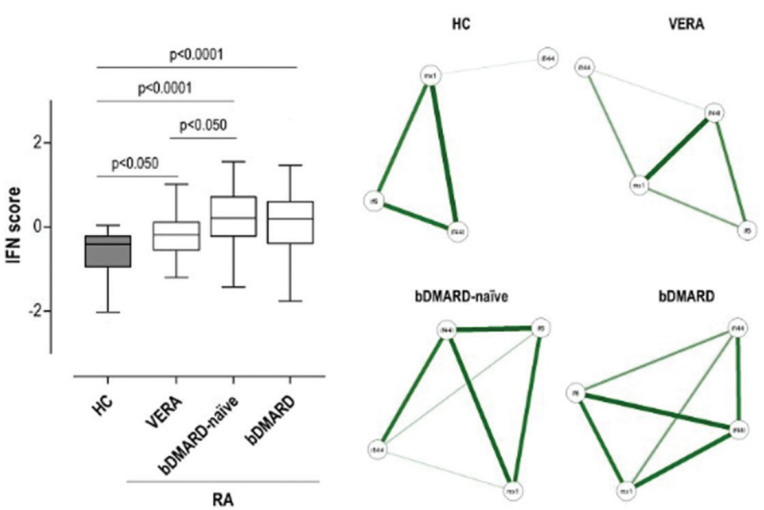

Abstract AB0041 - Figure 1

Conclusions: a certain heterogeneity within the IFN signature can be recognised in RA, depending on the clinical stage. The structure of the IFN signature may be a potential explanation for the controversy in this field and may represent a limitation for its use as a clinical biomarker.

Disclosure of Interest: None declared

DOI: 10.1136/annrheumdis-2018-eular.3195

\section{AB0042 ESSENTIAL OIL EXTRACTED FROM CHAMAECYPARIS OBTUSA ATTENUATES THE SEVERITY OF COLLAGEN- INDUCED ARTHRITIS}

J. Lee ${ }^{1}$, Y. Park ${ }^{2}$, C.-H. Yoon ${ }^{2} .{ }^{1}$ Division of Rheumatology, Department of Internal Medicine, College of Medicine, The Catholic University of Korea, Uijeongbu Saint Mary's Hospital; ${ }^{2}$ Division of Rheumatology, Department of Internal Medicine, College of Medicine, The Catholic University of Korea, Seoul, Korea., Seoul, Korea, Republic of Ireland

Background : Chamaecyparis obtusa (C. obtusa) is a tropical tree species found in Japan and the southern region of South Korea. Although the biological activities of essential oil extracted from C. obtusa (EOCO) are not yet fully understood, essential oil has been shown to have antibacterial activities and anti-inflammatory effects.
Objectives: We investigated the effect of essential oil extracted from Chamaecyparis obtusa on the development of collagen-induced arthritis $(\mathrm{CIA})$, a well-characterised preclinical model of human RA.

Methods: DBA1/J mice were injected intraperitoneally with EOCO dissolved in dimethyl sulfoxide (DMSO) 3 times a week for 5 weeks after type II collagen (CII) immunisation. Arthritis severity was evaluated by clinical and histological parameters. Serum anti-CII antibody levels were assayed by ELISA. linterleukin-17 (IL17) expressions in the joints were determined by immunohistochemistry. Effect of EOCO on Th17 cells and natural killer (NK) cells was analysed using flow cytometry.

Results: EOCO markedly reduced the clinical and histological severity of CIA Serum anti-CII antibody levels and the expression of IL-17 in the joints were significantly lower in mice treated with EOCO than those treated with DMSO alone. EOCO inhibited the differentiation of Th17 cells in total splenocytes, not in isolated $\mathrm{CD}^{+} \mathrm{T}$ cells, from mice. EOCO increased the production of granzyme $\mathrm{B}$ and perforin in NK cells.

Conclusions: Our data demonstrate a protective effect of EOCO on the development of CIA through the inhibition of Th17 cell differentiation. This effect may be related with NK cell activity enhanced by EOCO.

\section{REFERENCES:}

[1] Hong EJ, Na KJ, Choi IG, Choi KC, Jeung EB. Antibacterial and antifungal effects of essential oils from coniferous trees. Biol Pharm Bul 2004;27:863-6.

[2] An BS, Kang JH, Yang H, Jung EM, Kang HS, Choi IG, et al. Anti-inflammatory effects of essentia oils from Chamaecyparis obtusa via the cyclooxygenase-2 pathway in rats. Mol Med Rep 2013;8:255-9.

Acknowledgements: This study was carried out with the support of 'Forest Science and Technology Projects (Project No. S111114L020100)' provided by Korea Forest Service.

Disclosure of Interest: None declared

DOI: 10.1136/annrheumdis-2018-eular.1413

\section{AB0043 1 THE ELASTICITY PROPERTIES OF PROBIOTIC BACTERIA WALL ASSOCIATED WITH BENEFICIAL MODULATORY ACTIVITY ON INNATE IMMUNITY OF THE HOST}

L.P. Babenko, R.V. Bubnov, L.M. Lazarenko, M.Y. Spivak. Interferon, Zabolotny Institute of Microbiology and Virology, National Academy of Sciences of Ukraine, Kyiv, Ukraine

Background: Probiotics have tremendous potential to develop healthy diets and integrated approach for immunity-related diseases treatment and prevention; $;^{1,2}$ are effective actors in distant sites $^{3}$ with strong potential for applications in rheumatology. The cell wall of probiotic bacteria plays an essential role in many aspects of modulating beneficial immune response; ${ }^{4}$ its elasticity properties associated with probiotic beneficial effects and can warrant to stratify strains on their modulatory activity on innate immunity to justify individualised and personalised approach for nutrition and prevention.

Objectives: The aim was to study the effect of lactic acid bacteria (LAB) and bifidobacteria strains on phagocytic system cells functional activity and immunoregu latory cytokines synthesis in vitro in regards to the bacteria surface properties as cell walls elasticity using atomic force microscopy (AFM).

Methods: We conducted experimental studies on BALB/c line mice 18-20 g weight using lyophilized strains of LAB - Lactobacillus acidophilus IMV B-7279, L. casei IMV B-7280, L. delbrueckii subsp. bulgaricus IMV B-7281 and bifidobacteria - Bifidobacterium animalis VKL, B. animalis VKB. We cultivated the macrophages received from the peritoneal cavity of mice by common method individually with the strains of $L A B$ and bifidobacteria. We estimated the impact of $L A B$ and bifidobacteria strains on the functional activity of peritoneal cavity macrophages using the conventional methods of study oxygen-dependent bactericidal activity, nitric oxide production, their effect on the immunoregulatory cytokines. We used AFM scanning to estimate bacteria cell walls elasticity.

Results: All strains demonstrated a stimulating effect on the functional activity of macrophages and ability to produce $\mathrm{NO} / \mathrm{NO} 2$ in vitro. Lactobacilli strains increased the production of IL-12 and IFN- $\gamma$ in vitro. The AFM demonstrated different degree of the cell walls elasticity in various strains of $L A B$ and bifidobacteria. Among lactobacilli the most elastic cell wall was found in L. delbrueckii subsp. bulgaricus IMV B-7281, and among bifidobacteria - in B. animalis VKL, which induced the considerable activation of the phagocytes. Probiotic strains survival in 